\title{
Effects of resveratrol and alcohol on mood and cognitive function in older individuals
}

\author{
Andrew Scholey ${ }^{\mathrm{a}, *}$, Sarah Benson ${ }^{\mathrm{a}}$, Con Stough ${ }^{\mathrm{a}}$ and Creina Stockley ${ }^{\mathrm{b}}$ \\ ${ }^{a}$ Centre for Human Psychopharmacology, Swinburne University, Melbourne, VIC, Australia \\ ${ }^{\mathrm{b}}$ Australian Wine Research Institute, Glen Osmond, SA, Australia
}

\begin{abstract}
.
BACKGROUND: Heavy consumption of alcohol has well-documented negative effects on cognition both acutely and chronically. Nevertheless there is increasing research interest in the potential positive influence on cognitive functioning of bioactive dietary components including resveratrol from red wine.

OBJECTIVE: This study aimed to compare the acute mood and cognitive effects of resveratrol-enriched red wine with red wine alone during mentally effortful tasks in older individuals.

METHODS: Following a double-blind, balanced, crossover design, 16 volunteers (mean age 70.44 years) were tested on two occasions, once following $100 \mathrm{ml}$ red wine; once following the same beverage but enriched with $200 \mathrm{mg}$ resveratrol. They completed six cycles of the Cognitive Demand Battery comprising Serial Threes, Serial Sevens, a Rapid Visual Information Processing task and ratings of mental fatigue. Serum resveratrol and metabolites were co-monitored.

RESULTS: There were differential cognitive effects of the two treatments. Compared with red wine alone, resveratrol-enriched wine was associated with significantly enhanced Serial Sevens performance. Conversely red wine alone resulted in better performance during Serial Threes. Serum analysis confirmed absorption of resveratrol metabolites.

CONCLUSIONS: These results may reflect increased blood flow from resveratrol and the documented stimulant effects of alcohol at low levels. However the study requires replication with an inert control group.
\end{abstract}

Keywords: Resveratrol, cognition, ageing, polyphenols

\section{Introduction}

Cognitive function refers to mental processes by which knowledge is acquired and manipulated. The term encompasses perception, attention, working memory, secondary memory and executive functioning. Dementia is a debilitating form of cognitive dysfunction and, while there are other contributing factors, age is by far the most important risk factor for dementia and mild cognitive impairment (MCI) which can be prodromal for Alzheimer's disease (AD), the most common form of dementia. Globally, the population aged 60 years of age is projected to triple by

${ }^{*}$ Corresponding author: Professor Andrew Scholey, Centre for Human Psychopharmacology, Swinburne University, Melbourne, VIC 3122, Australia. Tel.: +6103 9214 8932; E-mail: andrew@ scholeylab.com.
2050, while the those aged over 80 years is projected to increase by five-fold [1]. At present there are few options for the treatment of cognitive dysfunction and dementia. The cholinesterase inhibitors (e.g. Aricept) used in AD treatment may be effective in delaying disease progression in some patients but they are often poorly tolerated and effective only at certain stages of the disease. This has led to the search for candidate lifestyle factors, including exercise and diet, which may positively modify the progression of cognitive ageing and AD.

High levels of alcohol consumption results in impaired cognitive functions both acutely [2] and with chronic use [3]. In the case of the latter, long-term intake of high levels of alcohol can lead to severe impairment and structural brain damage [4]. Paradoxically, the past 15 years has seen evidence suggesting 
that there may be a j-shaped relationship between the consumption of alcoholic beverages, including wine, and cognitive function [5-11]. This suggests that low levels of alcohol consumption may offer a protective effect against age-related cognitive decline and associated disorders.

A 16-year longitudinal study conducted in Dubbo, New South Wales of 2805 men and women aged 60 years and older, suggested that any consumption of alcohol reduced the risk of developing dementia by $34 \%$ [12], and a similar Italian study of 15,807 patients suggested that less than $40 \mathrm{~g}$ alcohol as wine per day for women and less than $80 \mathrm{~g}$ for men is associated with a reduced risk of cognitive dysfunction including Alzheimer's disease [13]. For both men and women, compared to abstainers current moderate as well as cumulative lifetime alcohol consumption have been associated with better cognitive function, including processing and psychomotor speed, immediate and delayed recall, recognition memory, figural memory and working memory [14-17]. It should be noted however that such findings have not gone unchallenged in other contexts [18].

In addition to alcohol, wine contains phenolic compounds similar to those in fruits, vegetables and teas, the consumption of which has also been associated with a lower incidence of both mild cognitive dysfunction and dementias as well as other cerebrovascular diseases $[19,20]$. Rat in vivo studies have shown that the wine-derived phenolic compound, resveratrol, can preserve brain function in aged rats [21, 22]. Resveratrol, in combination with other wine-derived phenolic compounds, has also been shown in vivo to increase cerebral arteriole dilation in rats [23] and improve postischaemic cerebral perfusion after both acute [24] and chronic [25] dosing.

The acute neural effects of resveratrol were confirmed in a placebo-controlled study in young healthy human adults which showed that a single oral dose of $250 \mathrm{mg}$ and $500 \mathrm{mg}$ resveratrol increased blood flow to the brain while participants performed effortful cognitive tasks [26]. Cognitive function was not affected, however, although resveratrol metabolites were present in plasma throughout the cognitive task period. It is possible that the effects of resveratrol may be more pronounced in older individuals where cognitive functioning tends to be worse. Additionally the previous acute study used encapsulated resveratrol and it may be that other phenolics present in wine may potentiate any effect of resveratrol.
We therefore conducted a small pilot trial to compare the acute cognitive effects of a moderate amount of resveratrol-enhanced red wine, with red wine alone on cognitive performance in older adults. Serum resveratrol and metabolites were con-monitored to evaluate bioavailability of resveratrol administered in red wine.

\section{Materials and methods}

\subsection{Design}

The present study investigated the effects of a single moderate dose of resveratrol-enhanced red wine on mood and cognitive performance in older adults using a controlled, double-blind, crossover design. The study was approved by the Swinburne University Human Research Ethics Committee and conducted in accordance with the Declaration of Helsinki.

\subsection{Participants}

Ten males and six females with a mean age of 70.44 years $(S D=4.37$; range $=65-78$ years $)$ volunteered to participate in the study. Recruitment was accomplished via University and local community advertising and word of mouth. All subjects provided informed consent prior to participating and confirmed that they were in good health, non-smokers and not taking any medications (other than those to treat benign conditions).

\subsection{Treatments}

The treatments consisted of $100 \mathrm{ml}$ red wine (Jacob's Creek 2007 vintage Shiraz), the resveratrol treatment comprised of the same drink enriched with $100 \mathrm{mg}$ grape-derived resveratrol. To ensure double-blinding, both treatments were identical in appearance and were dispensed by an uninvolved third party. Treatment bottles were stored upright in a cool and dark environment and were inverted for two minutes prior to dispensing.

\subsection{Cognitive testing}

A computerised version of the Cognitive Demand Battery (CDB) was employed to assess the impact of the treatment on continuous cognitive demand [27]. 
The battery comprised of a 2-min Serial Threes subtraction task, a 2-min Serial Sevens subtraction task and a 5-min version of the Rapid Visual Information Processing (RVIP) task. The battery was repeated 6 times over the course of an hour.

Serial Threes and Serial Sevens began with a standard instructions screen informing the participant to count backwards in threes/sevens from a given number, as quickly as possible and using the numeric keypad to enter each response. Participants were also verbally informed that if they make a mistake, they should continue subtracting from the most recent number entered. A random number between 800 and 999 was presented on the computer screen which was cleared by entry of the first response and from then on, each digit on screen was represented by an asterisk. The tasks were continuous for two minutes and were scored for total number of subtractions and number of errors made. Where incorrect responses were entered, subsequent responses were scored in relation to the most recent response.

RVIP entailed the participant monitoring a continuous series of digits for targets of three consecutive odd or even digits. The digits were presented at the rate of 100 per minute and the participant responded to the detection of the target string by pressing the 'space-bar' as quickly as possible. The task continued for five minutes and contained eight correct string targets. The task was scored for percentage of target strings correctly detected, average reaction time for correct detections and number of false alarms.

\subsection{Subjective mood}

At the beginning and end of the battery participants rated their mood using the Bond-Lader Visual Analogue Mood scales [28], which produce ratings of 'alert', 'calm' and 'content'. They also indicated on $100 \mathrm{~mm}$ lines how 'mentally fatigued' and 'stressed' they felt.

\subsection{Quantification of trans-resveratrol and its metabolites}

Analyses of the samples were undertaken by The Australian Wine Research Institute (AWRI) at the AWRI-Metabolomics Australia unit. Specifically the levels of the major metabolites Resveratrol4'-O-glucuronide, Resveratrol-3- $O$-glucuronide, Resveratrol-4'-O-sulfate and Resveratrol-3-O $O$-sulfate were determined as well as the parent molecule.

The samples were prepared and analysed based on the method reported by Boocock et al. [29]. Resveratrol and the four metabolites were extracted from the plasma samples using solid-phase (SPE) with Phenomenex Strata-X $33 \mu \mathrm{m} 85 \AA$ polymeric reverse phase $60 \mathrm{mg} / 3 \mathrm{~mL}$ cartridges. The mass spectral analysis was carried out using an Agilent 1290 Infinity LC coupled with Agilent 6490 Triple Quadrupole LC/MS. Solvent A was $5 \mathrm{mM}$ ammonium acetate, $2 \%$ isopropanol in Milli-Q water, $\mathrm{pH}$ adjusted to 5.4 with acetic acid and solvent B was $2 \%$ isopropanol in methanol. A gradient elution was used with a flow rate of $0.9 \mathrm{ml} / \mathrm{min}$ and injection volume of $5 \mu \mathrm{L}$. Resveratrol and the four metabolites were detected in negative ion mode using electrospray ionisation measuring $10 \mathrm{MRM}$ transitions. The collision energy and source conditions were optimised for each metabolite.

\subsection{Procedure}

All testing took place in a suite of dedicated laboratories at Swinburne University's Centre for Human Psychopharmacology where participants remained for the duration of the sessions. Participants were asked to fast from the night prior to testing and not to consume anything other than water. Participants were also asked to maintain a record of their food and drink intake for the 24 hours prior to the two testing sessions. At baseline, participants completed two repetitions of the 10-min CDB. The first was to eliminate practice effects while the second constituted the baseline assessment. A fasting blood sample was then taken before participants were given the day's treatment which was consumed within a five minute period. At 45 minutes post treatment, a second blood sample was taken and, beginning $60 \mathrm{~min}$ post-ingestion a 60-minute battery comprising of six repetitions of the CDB was completed. The battery was interrupted following three repetitions in order to take a third 10-ml blood sample. On completion of the battery, a fourth blood sample was collected. Following a washout period of not less than $48 \mathrm{~h}$ the participant returned and repeated the procedure in the alternate treatment condition. Order of treatments was randomised and balanced. 


\subsection{Statistical analysis}

All analyses were performed using IBM SPSS Version 21. Prior to analysis of treatment effects, baseline scores for each outcome were analysed by one-way, repeated measures ANOVA to ensure that treatment effects were not confounded by chance differences in baseline scores. No baseline score differences approached statistical significance.

Using change-from-baseline scores, primary analysis of each outcome was undertaken using a 2-way repeated measures ANOVA incorporating treatment (resveratrol-enhanced red wine, red wine alone) $\times$ time (change from baseline at each cycle). Any significant main effects of treatment or treatment $\times$ time interactions were further investigated using paired samples $t$-tests comparing scores between treatments at each cycle of the CDB. All testing was two-tailed and comparisons were planned prior to testing.

\section{Results}

\subsection{Cognitive testing}

There were differential effects of the two treatment effects on both Serial Threes and Serial Sevens (Fig. 1). The was a significant effect of Treatment on Serial Threes, $\mathrm{F}(1,11)=14.29, p=0.004$, with participants making more responses in the Red wine condition than with resveratrol. Planned comparisons at each time point revealed that these effects reached significance at cycle $5(t(15)=2.23, p=0.041)$ and cycles $6(t(15)=2.95, p=0.01)$. For Serial Sevens there was a significant treatment effect, $\mathrm{F}(1,10)=9.95$, $p=0.009$, with more responses in the Resveratrol than the Red Wine condition, at individual time points, these effects were significant during the second $(t(15)=3.51$, $p=0.003)$ and fourth $(t(15)=3.08, p=0.008)$ cycles. There were no other significant effects.

\subsection{Quantification of trans-resveratrol and its metabolites}

Analysis was performed only on the serum derived from the blood sample drawn at the halfway point of the cognitive battery. Analysis of serum samples revealed that resveratrol and its metabolites were absorbed (Fig. 2). Following the red wine treatment no

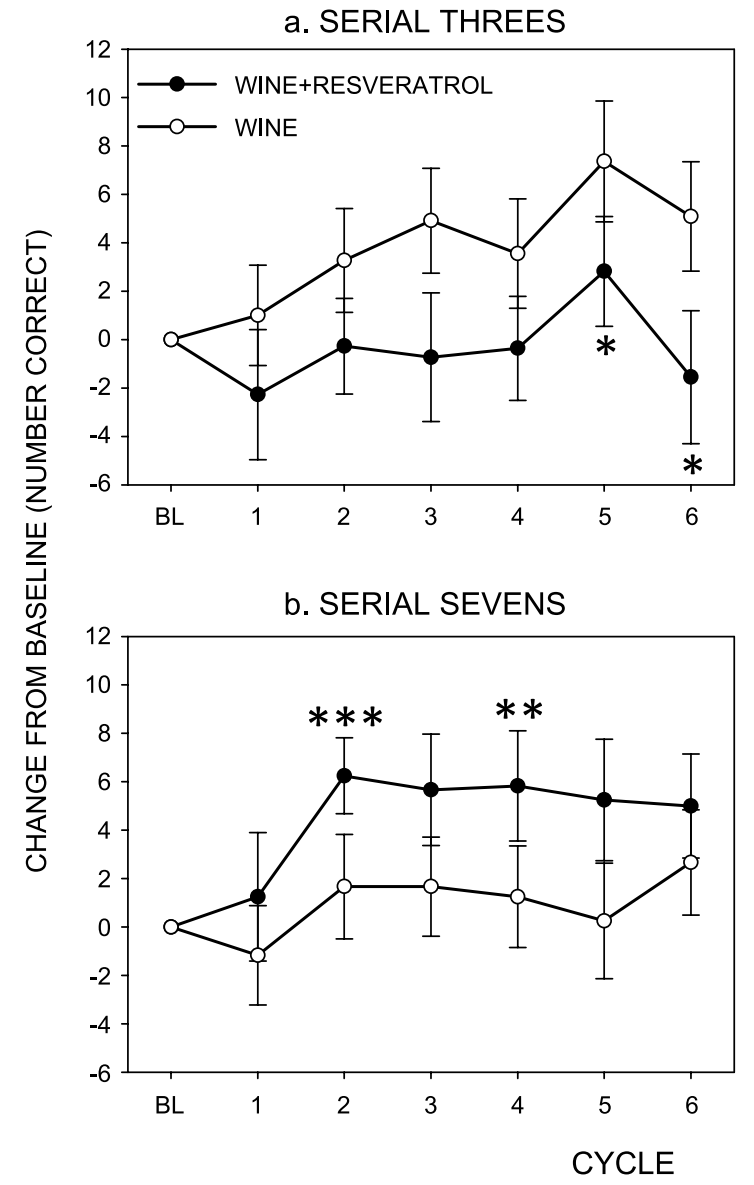

Fig. 1. Effects of red wine with and without resveratrol on Serial Subtractions. Graphs depict means (with SEMs) change-frombaseline correct responses for (a) Serial Threes and (b) Serial Sevens over 6 cycles of the Cognitive Demand Battery $\left({ }^{*} p<0.05\right.$; ${ }^{* *} p<0.01$; ${ }^{* * *} p<0.005$ at the time point indicated).

metabolites were detected, whereas resveratrol administration resulted in detectable levels of Resveratrol-4' $O$-glucuronide, Resveratrol-3'-O-glucuronide and Resveratrol-3'-O-sulfate. Resveratrol-4'-O-sulfate levels were negligible in both treatments. Regarding the parent molecule, there were small amounts present in the red wine condition but these were higher in the resveratrol condition.

\section{Discussion}

These data show that, compared with red wine alone, resveratrol-enriched red wine improves performance during a cognitively demanding cognitive battery, 


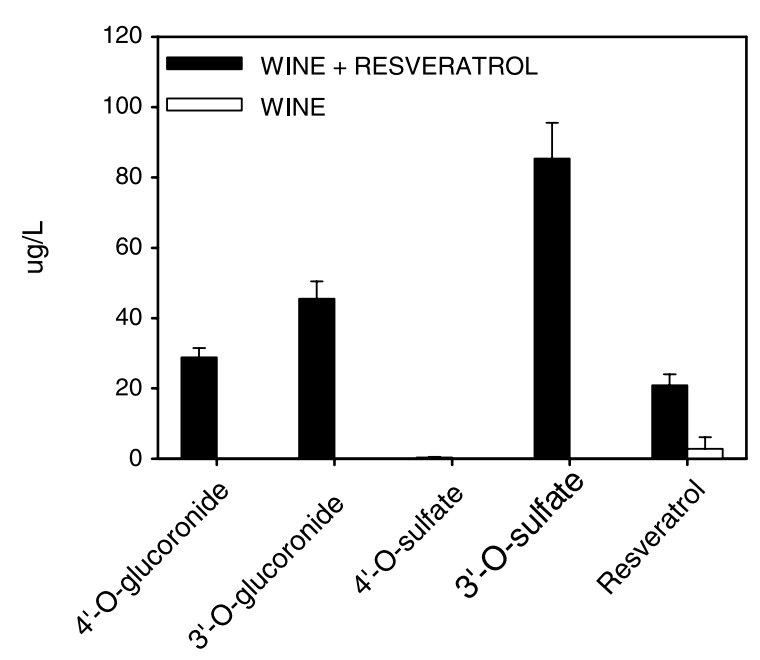

Fig. 2. Levels of resveratrol metabolites and the parent molecule in serum from blood drawn during the Cognitive Demand Battery.

specifically targeting the more effortful Serial Sevens. Conversely, the less effortful Serial Threes was improved in the Red wine alone condition compared with resveratrol.

Given that there was no inert control, these results should be interpreted with caution. However it is possible that the effects on Serial Sevens may be attributable to the acute effects of resveratrol on cerebral blood flow [26]. It has previously been shown that interventions which increase blood flow to the brain, including cocoa flavanols, can increase performance on this battery [30].

Regarding the bioavailability of resveratrol, levels of the major metabolites were consistent with previously reported data. With respect to the parent molecule, there was some evidence that both treatments led to detectable levels of serum resveratrol. Australian Shiraz can contain relatively high levels of the molecule [31], these levels, however, are far lower than those in the enriched wine. Inspection of the data revealed that the values seen here were the result of resveratrol being detected in one individual only following red wine alone. Thus it maybe that this result is due to a measurement error although this is consistent with unpublished in-house data showing resveratrol being detected in approximately 5-10\% of subjects following $200 \mathrm{ml}$ red wine. Indeed other than this one data point, all measures were below the threshold of detection in the red wine alone condition. The negligible levels of resveratrol metabolites in the control condition confirm that the washout period was adequate.
We have previously argued that interventions which increase the delivery and/or utilisation of metabolic resources are candidate cognitive enhancers [2, 27, 30]. While this may be the mechanisms underlying the effects seen here, it is worth noting that increased blood flow resulting from polyphenol administration can occur in the absence of measureable cognitive enhancement [26, 32].

An obvious alternative explanation is that the difference seen on Serial Sevens performance represent a negative effect of alcohol alone on performance of this effortful task. This is unlikely, however, as both drinks contained the same level of alcohol, although it is possible that resveratrol negated any impairing effects of alcohol. Additionally, the level of alcohol was relatively low (11-12g). It is know that at lower levels, and during the rising limb of the blood alcohol curve, alcohol acts as a stimulant and can improve elements of cognitive function [33]. Indeed these properties may underlie the superior performance in the alcohol alone condition during Serial Threes. This task relies more on psychomotor speed and attention than Serial Sevens so is likely to be susceptible to the stimulant effects of low dose alcohol. It appears that any such benefits to performance of Serial Threes is reversed by the addition of resveratrol.

In conclusion, this small pilot study indicates that acute administration of resveratrol-enriched red wine produces a different cognitive profile to that of red wine alone. These results suggest that resveratrol is centrally active. Clearly the study needs to be repeated in a larger cohort and with a de-alcoholised wine or other, psychopharmacologically inert control.

\section{References}

[1] Kelner KL, Marx J. Patterns of aging. Science. 1996;273:41.

[2] Scholey A, Benson S, Neale C, Owen L, Tiplady B. Neurocognitive and mood effects of alcohol in a naturalistic setting. Human Psychopharmacology: Clinical and Experimental. 2012;27(5):514-6.

[3] Ling J, Heffernan TM, Buchanan T, Rodgers J, Scholey AB, Parrott AC. Effects of alcohol on subjective ratings of prospective and everyday memory deficits. Alcoholism: Clinical and Experimental Research. 2003;27(6):970-4.

[4] Harper C. The neuropathology of alcohol-related brain damage. Alcohol and Alcoholism. 2009;44(2):136-40.

[5] Deng J, Zhou DH, Li J, Wang YJ, Gao C, Chen Me. A 2-year follow-up study of alcohol consumption and risk of dementia. Clinical neurology and neurosurgery. 2006;108(4): 378-83. 
[6] Ganguli M, Vander Bilt J, Saxton J, Shen C, Dodge H. Alcohol consumption and cognitive function in late life A longitudinal community study. Neurology. 2005;65(8):1210-7.

[7] Larrieu S, Letenneur L, Helmer C, Dartigues J, BarbergerGateau P. Nutritional factors and risk of incident dementia in the PAQUID longitudinal cohort. Journal of Nutrition, Health \& Aging. 2004.

[8] Mehlig K, Skoog I, Guo X, Schütze M, Gustafson D, Waern $\mathrm{M}$, et al. Alcoholic beverages and incidence of dementia: 34-year follow-up of the prospective population study of women in Göteborg. American journal of epidemiology. 2008;167(6):684-91.

[9] Mukamal KJ, Kuller LH, Fitzpatrick AL, Longstreth Jr W, Mittleman MA, Siscovick DS. Prospective study of alcohol consumption and risk of dementia in older adults. JAMA: The journal of the American Medical Association. 2003;289(11):1405-13.

[10] Reid M, Van Ness PH, Hawkins KA, Towle V, Concato J, Guo $\mathrm{Z}$. Light to moderate alcohol consumption is associated with better cognitive function among older male veterans receiving primary care. Journal of geriatric psychiatry and neurology. 2006;19(2):98-105.

[11] Ruitenberg A, van Swieten JC, Witteman J, Mehta KM, van Duijn CM, Hofman A, et al. Alcohol consumption and risk of dementia: The Rotterdam Study. The Lancet. 2002;359(9303):281-6

[12] Simons LA, McCallum J, Friedlander Y, Ortiz M, Simons J. Moderate alcohol intake is associated with survival in the elderly: The Dubbo Study. Medical Journal of Australia. 2000;173(3):121-4.

[13] Zuccalà G, Onder G, Pedone C, Cesari M, Landi F, Bernabei R, et al. Dose-Related Impact of Alcohol Consumption on Cognitive Function in Advanced Age: Results of a Multicenter Survey. Alcoholism: Clinical and Experimental Research. 2001;25(12):1743-8.

[14] Bond GE, Burr RL, McCurry SM, Rice MM, Borenstein AR, Larson EB. Alcohol and cognitive performance: A longitudinal study of older Japanese Americans. The Kame Project. International psychogeriatrics / IPA. 2005;17(4):653-68.

[15] Stampfer MJ, Kang JH, Chen J, Cherry R, Grodstein F. Effects of moderate alcohol consumption on cognitive function in women. The New England journal of medicine. 2005;352(3):245-53.

[16] Cho E, Hankinson SE, Willett WC, Stampfer MJ, Spiegelman D, Speizer FE, et al. Prospective Study of Alcohol Consumption and the Risk of Age-Related Macular Degeneration. Archives of Ophthalmology. 2000;118(5):681-8.

[17] Espeland MA, Coker LH, Wallace R, Rapp SR, Resnick $\mathrm{SM}$, Limacher $\mathrm{M}$, et al. Association between alcohol intake and domain-specific cognitive function in older women. Neuroepidemiology. 2006;27(1):1-12. PubMed PMID: ISI:000239243700001.

[18] Fillmore KM, Kerr WC, Stockwell T, Chikritzhs T, Bostrom A. Moderate alcohol use and reduced mortality risk: Systematic error in prospective studies. Addiction Research \& Theory. 2006;14(2):101-32.

[19] Frisardi V, Panza F, Seripa D, Imbimbo BP, Vendemiale G, Pilotto A, et al. Nutraceutical Properties of Mediterranean Diet and Cognitive Decline: Possible Underlying Mechanisms.
Journal of Alzheimers Disease. 2010;22(3):715-40. PubMed PMID: ISI:000284755600002.

[20] Solfrizzi V, Panza F, Frisardi V, Seripa D, Logroscino G, Imbimbo BP, et al. Diet and Alzheimer's disease risk factors or prevention: The current evidence. Expert Review of Neurotherapeutics. 2011 May;11(5):677-708. PubMed PMID: ISI:000290834200014.

[21] Joseph JA, Fisher DR, Cheng V, Rimando AM, Shukitt-Hale B. Cellular and behavioral effects of stilbene resveratrol analogues: Implications for reducing the deleterious effects of aging. Journal of agricultural and food chemistry. 2008;56(22):10544-51.

[22] Oomen CA, Farkas E, Roman V, Van Der Beek EM, Luiten PG, Meerlo P. Resveratrol preserves cerebrovascular density and cognitive function in aging mice. Frontiers in aging neuroscience. 2009;1.

[23] Chan S-L, Capdeville-Atkinson C, Atkinson J. Red wine polyphenols improve endothelium-dependent dilation in rat cerebral arterioles. Journal of cardiovascular pharmacology. 2008;51(6):553-8

[24] Ritz M-F, Curin Y, Mendelowitsch A, Andriantsitohaina R. Acute treatment with red wine polyphenols protects from ischemia-induced excitotoxicity, energy failure and oxidative stress in rats. Brain research. 2008;1239:226-34.

[25] Ritz M-F, Ratajczak P, Curin Y, Cam E, Mendelowitsch A, Pinet $\mathrm{F}$, et al. Chronic treatment with red wine polyphenol compounds mediates neuroprotection in a rat model of ischemic cerebral stroke. The Journal of nutrition. 2008;138(3):519-25.

[26] Kennedy DO, Wightman EL, Reay JL, Lietz G, Okello EJ, Wilde A, et al. Effects of resveratrol on cerebral blood flow variables and cognitive performance in humans: A double-blind, placebo-controlled, crossover investigation. The American journal of clinical nutrition. 2010;91(6):1590-7.

[27] Scholey AB, Harper S, Kennedy DO. Cognitive demand and blood glucose. Physiology \& behavior. 2001;73(4):585-92.

[28] Bond A, Lader M. The use of analogue scales in rating subjective feelings. British Journal of Medical Psychology. 1974;47(3):211-8

[29] Boocock DJ, Patel KR, Faust GE, Normolle DP, Marczylo TH, Crowell JA, et al. Quantitation of trans-resveratrol and detection of its metabolites in human plasma and urine by high performance liquid chromatography. Journal of Chromatography B. 2007;848(2):182-7.

[30] Scholey AB, French SJ, Morris PJ, Kennedy DO, Milne AL, Haskell CF. Consumption of cocoa flavanols results in acute improvements in mood and cognitive performance during sustained mental effort. Journal of Psychopharmacology. 2010;24(10):1505-14.

[31] Gu X, Creasy L, Kester A, Zeece M. Capillary electrophoretic determination of resveratrol in wines. Journal of agricultural and food chemistry. 1999;47(8):3223-7.

[32] Wightman EL, Haskell CF, Forster JS, Veasey RC, Kennedy DO. Epigallocatechin gallate, cerebral blood flow parameters, cognitive performance and mood in healthy humans: A double-blind, placebo-controlled, crossover investigation. Human Psychopharmacology: Clinical and Experimental. 2012;27(2):177-86.

[33] Scholey AB, Fowles KA. Retrograde enhancement of kinesthetic memory by alcohol and by glucose. Neurobiology of learning and memory. 2002;78(2):477-83. 